\title{
Binocular summation in detection of contrast flashes
}

\author{
ALEXANDER I. COGAN, GERALD SILVERMAN, and ROBERT SEKULER \\ Smith-Kettlewell Institute of Visual Sciences, San Francisco, California
}

\begin{abstract}
We studied monocular and binocular detection of foveal flashes of different contrast. When background contours were binocularly fused, detectability (d') of binocular test flashes was, on the average, twice the detectability of monocularly presented flashes. The precise amount of binocular advantage varied with test contrast: binocular improvement exceeded full summation for low test contrast, but fell below full summation at higher test contrasts. In the absence of contours in one eye, background luminances are not expected to sum, yet binocular detection is an average of $41.5 \%$ better than monocular detection. This indicates a difference in the functional organization of the fused binocular channel and a monocular channel.
\end{abstract}

Detectability of a local luminance increment flashed against a luminous background (a "contrast flash") depends on contrast sensitivity in a fundamental way, as expressed, for instance, by Weber's law $(\Delta I / I=$ const).

Sensitivity increases when observation is binocular rather than monocular. The nature of binocular improvement in sensitivity is not known, nor has its magnitude been unequivocally established. Neurophysiological data from simple cells in cat visual cortex has revealed true binocular facilitation, a response in excess of the sum of separate monocular responses (e.g., Burns \& Pritchard, 1968; Nelson, Kato, \& Bishop, 1977). However, in human visual psychophysics, such binocular facilitation is unknown. ${ }^{1}$

The magnitude of binocular improvement in sensitivity varies considerably among experimental situations (reviewed by Blake \& Fox, 1973). To summarize the range of findings, it is convenient to define sensitivity by the reciprocal of threshold contrast. Using $S_{B}, S_{R}$, and $S_{L}$ to denote sensitivity with both eyes, with the right eye alone, and with the left eye alone, respectively, and disregarding eye dominance,

$$
\left[\left(S_{R}+S_{L}\right) / 2\right]<S_{B}<\left(S_{R}+S_{L}\right) \text {. }
$$

The right member in the above expression indicates the upper limit for binocular improvement: "full summation." An example is provided by the case in which binocular detection of luminance increments is about twice as good as average monocular detec-

This work was supported by National Eye Institute Grants RO1 EYO 1558 and P30 1668 and by the Smith-Kettlewell Eye Research Foundation. R. Sekuler was on leave from Northwestern University. His participation in this research was made possible by a grant from the National Institute of Aging, AG-1251. Send reprint requests to A. I. Cogan, Smith-Kettlewell Institute of Visual Sciences, 2232 Webster Street, San Francisco, California 94115. tion (e.g., Kristofferson, cited in Green \& Swets, 1966). A special case is energy summation. Full energy summation is said to occur if one can take a monocular stimulus that is at threshold, divide its energy between the two eyes yet still keep the stimulus at threshold (Cohn \& Lasley, 1976). In fact, binocular improvement at absolute threshold falls short of full energy summation; at best, the binocular advantage is on the order of $70 \%-80 \%$ (Thorn \& Boynton, 1974). At its poorest, binocular performance was found to be only slightly better than monocular performance (e.g., Crawford, 1940).

There are various ways to explain intermediate degrees of binocular improvement. One approach is "probability summation" developed by Pirenne (1943). If one eye detects the stimulus with probability $P_{R}$, and the other eye with $P_{L}$, then both eyes will detect the stimulus with probability $P_{B}$, where

$$
P_{B}=1-\left(1-P_{R}\right)\left(1-P_{L}\right) \text {. }
$$

Campbell and Green (1965) offered a different, square-root summation model. They found that binocular thresholds for contrast detection were some 1.4 times lower than monocular thresholds (gratings with sine-wave luminance profile were the stimuli). Since the standard error of the sum of $n$ independent samples from a noisy process decreases as $21 / n$, an observer using two eyes could have a factor of 1.414 better sensitivity. Campbell and Green concluded that independent responses to contrast in the two eyes were combined to arrive at a binocular response to contrast at threshold; binocular sensitivity is better because two independent samples allow the observer to reduce the denominator of the signal/noise ratio upon which his decision depends.

The case for energy summation was strengthened by Thorn and Boynton (1974), who found absolute binocular thresholds a factor of 1.75 lower than 
monocular thresholds; they have argued convincingly that the improvement must be due to incomplete summation of monocular inputs.

Both square-root summation and probability summation models are two-stage models of physiological summation. ${ }^{2}$ In each, the outputs of two separate monocular detectors are combined to produce a common binocular output (Figure 1a). These models clearly differ from a one-stage summation model (Figure 1b) involving fusion of monocular inputs. This latter model can predict energy summation.

Binocular performance is significantly better when the monocular stimuli are simultaneous (Matin, 1962) and fall on corresponding retinal areas (e.g., Crozier \& Holway, 1938, 1939) than when either of these conditions is not fulfilled (Westendorf \& Fox, 1977). Yet, visibility of a target presented to one eye is not always affected by simultaneous stimulation of the corresponding retinal site in the opposite eye. In particular, the monocular channels seem to be independent when luminance, contour content (Bouman, 1955; Crawford, 1940), or contrast of the stimuli (Legge \& Rubin, 1981) is grossly different in the two eyes; perception then depends on the eye getting the stronger stimulus.

It appears that the visual system has more ways than one to process signals originating in each of the
A.

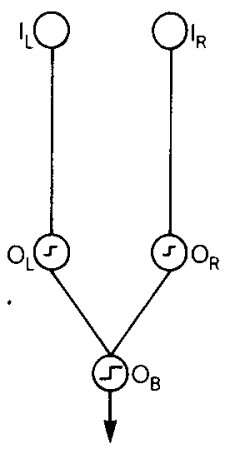

B.

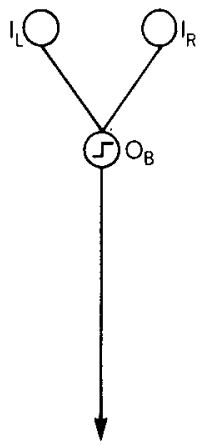

C.

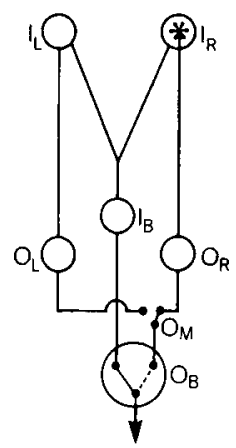

Figure 1. Schematic representation for different hypotheses on the organization of binocular summation. $I_{R}, I_{L}$, and $I_{B}$ are inputs-right, left, and binocular, respectively. $\hat{O}_{R}, O_{L}$, and $o_{B}$ are outputs-right, left, and binocular, respectively. Panel $A$ : Contrast is detected (step sign) in each monocular channel; then, the signals coming from two independent monocular detectors are combined; binocular detection is improved due to probability summation, or square-root summation. Panel B: Contrast is detected after monocular inputs are combined; binocular improvement is the result of energy summation. Panel C: Monocular inputs are combined as in $B$ when and only when they are matched; the "fused" input $I_{B}$ is then fed into the output generator $O_{B}$. The same generator may also receive a signal (produced by a flash, asterisk) from one monocular channel, "gated" by the switch $O_{M}$. The gate at $O_{M}$ is activated only by unmatched input. Interaction then occurs (dashed line) between the fused and the nonfused input entering the output generator $\mathrm{O}_{\mathrm{B}}$. The model implies that the final output may be produced by the fused channel only, by one monocular channel, or by weighted contributions from these channels. two eyes. One of us (Cogan, 1982) proposed that full summation (fusion) of monocular inputs occurs in an exclusively binocular branch of the system, which processes matched contours on corresponding retinal areas. Unmatched contours are processed in a separate, interocular branch of the system. This model is presented in Figure 1c. The diagram contains a channel for energy summation, the same as in Figure $1 \mathrm{~b}$, but this channel is activated only when the contours present in the two eyes are matched (fused); a different, interocular channel functions in the presence of unmatched contours such that the eye with the stronger contours gets through, cutting off the other monocular channel. The final output may be produced by the fused channel and one monocular channel, for example, when a contrast flash is delivered to one eye. According to the model, binocular summation of background luminance occurs within the area delimited by the fused background contours. When brief monocularly presented flashes appear inside this area, their detectability is depressed in proportion to the increase in adaptation state created by binocular summation of background luminance. But the situation is complicated by the effect of contrast flashes themselves: monocularly presented flashes act as unmatched contours, and thus may disrupt fusion of the background contours.

The model in Figure 1c predicts that the amount of binocular improvement in detectability of local luminance increments will vary inversely with testflash contrast. Weak monocular flashes would leave the fused channel in operation; therefore, the increment presented monocularly would be subthreshold against binocularly summed background luminance (while the same increment will be suprathreshold when given to both eyes simultaneously). Strong flashes will cause disruption of fusion and thus decrease binocular summation of background luminance.

Our experiments show that the prediction is fulfilled. With fused background contours, binocular facilitation (improvement in excess of full summation) occurs for the detection of weak flashes. But as the increment flash grows, the amount of binocular improvement decreases.

\section{GENERAL METHOD}

Observers had to detect local luminance increments within the central area of the binocular visual field, against a luminous background delimited by clearly visible contours. The background contours were binocularly fused. In different series, the binocular background luminance, and thus the test contrast, was varied. With flashes presented either to one eye or to both eyes simultaneously, we compared detection for binocular and monocular test conditions, for a range of test-contrast values.

\section{Apparatus}

Targets and test stimuli (Figure 2) were presented in a mirror stereoscope with a $228-\mathrm{cm}$ arm length. The targets were identical for both eyes, except for a "nonius mark"-a vertical line-in the upper periphery of the left target and in the lower periphery 

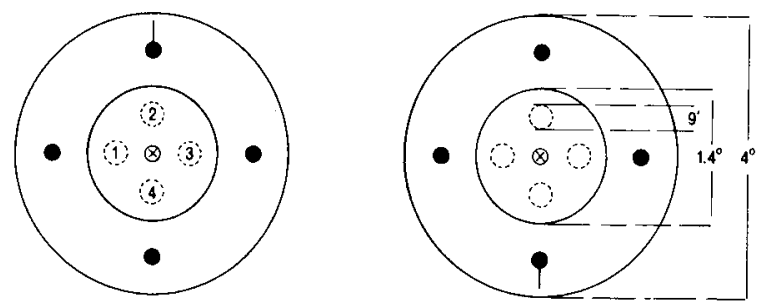

Figure 2. Diagram of the targets and stimuli locations. When targets were stereoscopically fused, the vertical segments appeared along the same vertical dimension. $X$ shows the position of the red fixation spot. Broken lines encircle separate LED loci (the LEDs were flashed from target rear); numerals denote the convention for locus identification.

of the right target; when the two targets were perfectly fused, the two segments were seen along the same central vertical meridian. Targets appeared against a matte white background, with the following details to aid fusion: $A$ black line ( 5 arc min thick) encircled the target center (1.4 deg diam); four black dots were positioned symmetrically outside the circle. The targets were frontilluminated with white light. With the help of a D/A converter and feedback from selenite photocells facing the lamps, a computer produced the desired target luminance in the range of 25 to $250 \mathrm{~cd} / \mathrm{m}^{2}$.

From the target rear, a red LED (Monsanto 5053, $660 \mathrm{~nm}$ ) produced a continuously illuminated fixation mark in the exact target center, and four yellow LEDs (Monsanto $5353,565 \mathrm{~nm}$ ) produced test flashes (170 msec duration) at four loci symmetrically positioned about the fixation light ( 22 arc min away from its center). Thus, all test stimuli were foveal, contained within an area of some $.9 \mathrm{deg}$ diam. Steady state luminances of the test stimuli were determined with a Pritchard Spectra Spot photometer. The same computer that controlled the luminance, also randomized and presented stimuli, recorded responses, and analyzed the data.

Contrast was defined as the Weber fraction $(\Delta I / I)$. With the luminance of the LEDs constant $\left(5 \mathrm{~cd} / \mathrm{m}^{2}\right)$, as the background luminance varied from 250 to $25 \mathrm{~cd} / \mathrm{m}^{2}$, contrast varied in the range of $2 \%$ to $20 \%$.

\section{Observers}

The authors were the observers. All had normal visual acuity, R.W.S. with hyperopic correction and A.I.C. with myopic correction, both on the order of $1.25 \mathrm{D}$ in spherical equivalent. Lenses to correct the ametropia were inserted in the trial frame before each experimental run. All observers had normal binocular vision (foveal fusion and stereopsis) and normal color vision.

\section{General Procedure}

Any observer's threshold varies from one day to the next, but we wished our forced-choice detection data to be as little affected as possible by such variation. To achieve this, we estimated each observer's threshold daily and then scaled all stimuli used that day for the observer relative to his threshold. For one observer (R.W.S.), who had had little previous experience with detection of luminance increments, thresholds were measured twice daily and stimuli scaled separately for his morning and afternoon forced-choice detection runs.

Thresholds were estimated as follows. Blocks of 12 trials were presented at various contrasts covering about a log unit range. On every trial, a randomly chosen pair of LEDs was flashed, and the observer was required to identify them. Seven contrast levels were tested, with a descending series followed by an ascending series. Thresholds were estimated by linear interpolation of the contrast at which performance just exceeded the guessing level $(8$ correct out of 12 presented; $\mathrm{p}<.05$; a full description is given in Cogan, 1982).

This procedure yielded monocular thresholds for each of the observer's eyes, and also binocular thresholds. To determine binocular thresholds, the same LEDs were presented to both eyes, at the same contrast. During all trials, the background targets and luminance were the same for both eyes. Monocular and binocular thresholds were taken in separate series, randomly ordered. Then followed the main procedure of forced-choice detection of contrast flashes. In different series, test contrast assumed different values between the binocular and the monocular threshold estimates. Further detail is given below.

\section{Threshold Estimates}

Binocular thresholds were a factor of 1.7 lower, on the average, than monocular thresholds. Table 1 shows individual observers' results, in terms of monocular and binocular sensitivities (reciprocals of contrast at threshold). Note that, for two observers, monocular sensitivity was very similar for both eyes, but that, for R.W.S., sensitivity was different for the two eyes. In addition, R.W.S. had generally lower sensitivity for the contrast flash than did the two other observers.

\section{EXPERIMENT 1: BINOCULAR IMPROVEMENT IN DETECTION OF CONTRAST FLASHES AS A FUNCTION OF TEST CONTRAST}

\section{Method}

Background luminance, and thus test contrast, was constant throughout an experimental series. On each self-initiated trial, the observer had to judge whether any signals were presented and, if so, at which loci. A series consisted of 160 trials: on 40 trials, one locus was flashed, with all four loci being equiprobable; on 60 trials, two loci were simultaneously flashed, with the six possible pair combinations being equiprobable; on 40 trials, three loci were flashed, with all trios being equiprobable; on 10 trials, all four loci were illuminated simultaneously; finally, during another 10 trials, no LEDs were flashed. Within each subblock of 40 trials, $0,1,2,3$, and 4 LEDs appeared equally often.

After initiating a trial with a buttonpress, the observer pushed the same button again if the response was "no flashes"; four other buttons corresponded to the loci at which flashes could be seen. No feedback was provided, but the observer was cued to onset of test trials by an auditory signal. The average duration of each series was about $15 \mathrm{~min}$. Within a set of three (e.g., right eye tested, left eye tested, binocular test), intervals between series were 5 to $10 \mathrm{~min}$ long. Intervals between sets varied from $1 / 2 \mathrm{~h}$ to several days. At the end of the experiment, a sufficient number of test contrast values was used to cover the range of $2.0>\mathrm{d}^{\prime}{ }_{\text {monoc }}$ $>0.0$. A particular contrast value for a given set was chosen in a pseudorandom fashion.

\section{Results}

The signal detectability theory parameter $\mathrm{d}^{\prime}$ (Swets, 1964) was used to evaluate performance from various

Table 1

Sensitivity for a Contrast Flash (Reciprocal of Contrast at Threshold)

\begin{tabular}{|c|c|c|c|c|c|c|c|}
\hline \multirow{3}{*}{$\begin{array}{c}\text { Ob- } \\
\text { server }\end{array}$} & \multicolumn{6}{|c|}{ Sensitivity } & \multirow[b]{3}{*}{$\mathrm{BI}^{*}$} \\
\hline & \multicolumn{2}{|c|}{$\mathrm{S}_{\mathrm{R}}$} & \multicolumn{2}{|c|}{$S_{L}$} & \multicolumn{2}{|c|}{$\mathrm{S}_{\mathrm{B}}$} & \\
\hline & Mean & SD & Mean & $\mathrm{SD}$ & Mean & SD & \\
\hline J.S. & 31.2 & 2.9 & 32.2 & 4.2 & 50.0 & 5.0 & 1.58 \\
\hline A.I.C. & 27.9 & 3.6 & 27.8 & 4.6 & 50.0 & 7.5 & 1.82 \\
\hline R.W.S. & 14.3 & 2.4 & 8.5 & 2.2 & 19.7 & 6.2 & 1.73 \\
\hline
\end{tabular}

*Binocular improvement, calculated as $S_{B} /\left[\left(S_{R}+S_{L}\right) / 2\right]$; overall mean $=1.71$. 
aspects of our data. For each block of 160 trials, we considered the four possible stimulus loci separately and then calculated $\mathrm{d}^{\prime}$ in the standard manner for a "yes-no" experiment. For each locus, we calculated the proportion of trials on which the stimulus was correctly reported (a "hit") and the proportion of trials, for that same locus, on which the stimulus was reported although it had not been presented (a "false alarm"). This pair of proportions allowed d' to be calculated from tabled values (Swets, 1964). We excluded from our analysis the trials on which either all the LEDs or none of the LEDs were flashed.

After estimating $\mathrm{d}^{\prime}$ for the four individual loci over a block of trials, we sorted and combined them in ways appropriate to our experimental questions. For example, the individual $d^{\prime}$ s for the four loci were combined to yield the mean d' for all trials in a block in which $n$ LEDs had been presented, where $n=1$, 2 , or 3. A variation of $d^{\prime}$ with the number of LEDs flashed simultaneously would signify nonindependence of the neighboring stimulation loci (e.g., spatial summation). Conversely, an absence of such variation would mean that signals from different loci in the same field were processed in separate spatial channels. In addition, mean $\mathrm{d}^{\prime}$ value was determined separately for trials following a presentation, and trials following omission of a stimulus at a given locus. A significant difference between these two $\mathrm{d}^{\prime}$ values would signify nonindependence of sequential trials. Finally, grand mean $d^{\prime}$ values obtained for each experimental series when $d^{\prime}$ 's were averaged over the four loci allow comparisons between performance of the eyes, among observers, and in different conditions.

Detection was independent of the number of loci stimulated simultaneously, since mean $\mathrm{d}^{\prime}$ remained essentially the same for trials in which 1,2 , or 3 LEDs were illuminated simultaneously (Figure 3 ). In particular, detection did not improve with the number of flashes given simultaneously. The four stimulation loci thus represented independent spatial channels.

At any LED locus, detection on any trial was largely independent of whether that LED had or had not been illuminated on the previous trial. Values of $d^{\prime}$ calculated for trials following stimulus omission did not significantly differ from $\mathrm{d}^{\prime}$ values calculated for trials following stimulus presentation. Mean $\mathrm{d}^{\prime}$ was as follows: (1) For all trials following stimulus omission, $\mathrm{d}_{\mathrm{B}}^{\prime}=2.14, \mathrm{~d}_{\mathrm{R}}^{\prime}=1.40$, and $\mathrm{d}_{\mathrm{L}}^{\prime}=1.46$; and (2) for trials following stimulus presentation, $d^{\prime}{ }_{B}=$ $2.05, \mathrm{~d}_{R}^{\prime}=1.34$, and $\mathrm{d}_{L}^{\prime}=1.31$. In only one comparison of the nine (Observer J.S., left eye) was mean $\mathrm{d}^{\prime}$ for trials following stimulus omission 1.21 times larger than mean $d^{\prime}$ for trials following stimulus presentation ( $p<.05$, $t$ test: repeated measures). The results, given in Table 2, do not bear out Collier's (1954) conclusion that the outcome of a psycho-
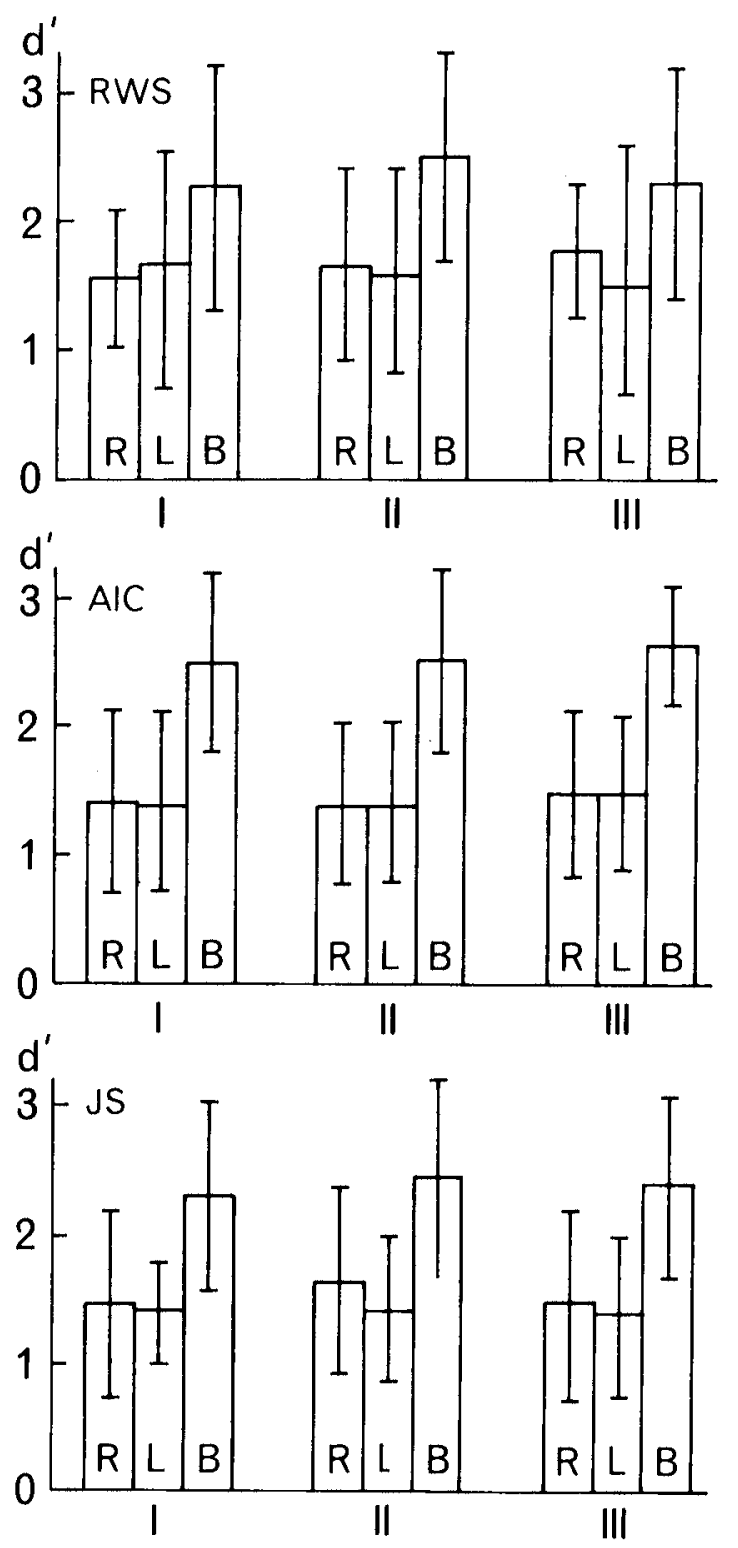

Figure 3. Detectability of a contrast flash as a function of the number of foveal loci stimulated simultaneously. Detection (mean $d^{\prime}$, ordinate) was essentially independent of the number of stimuli (I, II, or III) presented simultaneously. This was the case in the right monocular field (R), the left field (L), and the binocular field (B). Since d' does not change from I to III, there was no spatial interaction between the stimulation loci. (Note also the advantage in binocular performance. Here, the average amount of binocular improvement in performance equals $68 \%$. Each mean was obtained by collapsing data across all contrasts tested.)

physical trial is necessarily correlated with the outcome of the previous trial.

At all contrast values, binocular detection was superior to monocular detection. Mean binocular improvement $\left[d^{\prime}{ }_{B} / d^{\prime}{ }_{M}\right.$, where $d_{B}^{\prime}$ is the grand mean for binocular detection, and $d^{\prime}{ }_{M}$ is the average mon- 
Table 2

Mean $d^{\prime}$ for Trials Following Omission or Presentation of a Stimulus

\begin{tabular}{|c|c|c|c|c|c|c|c|c|c|c|c|c|}
\hline \multirow[b]{4}{*}{ Observer } & \multicolumn{12}{|c|}{ Testing Site } \\
\hline & \multicolumn{4}{|c|}{ Binocular } & \multicolumn{4}{|c|}{ Right Eye } & \multicolumn{4}{|c|}{ Left Eye } \\
\hline & \multicolumn{2}{|c|}{$00 \& 01$} & \multicolumn{2}{|c|}{$10 \& 11$} & \multicolumn{2}{|c|}{$00 \& 01$} & \multicolumn{2}{|c|}{$10 \& 11$} & \multicolumn{2}{|c|}{$00 \& 01$} & \multicolumn{2}{|c|}{$10 \& 11$} \\
\hline & Mean & SD & Mean & SD & Mean & SD & Mean & SD & Mean & SD & Mean & SD \\
\hline $\begin{array}{l}\text { J.S. } \\
\text { A.I.C. } \\
\text { R.W.S. }\end{array}$ & $\begin{array}{l}2.04 \\
2.13 \\
2.25\end{array}$ & $\begin{array}{l}.69 \\
.66 \\
.80\end{array}$ & $\begin{array}{l}1.92 \\
2.01 \\
2.21\end{array}$ & $\begin{array}{l}.61 \\
.70 \\
.77\end{array}$ & $\begin{array}{l}1.23 \\
1.35 \\
1.61\end{array}$ & $\begin{array}{l}.77 \\
.67 \\
.63\end{array}$ & $\begin{array}{l}1.24 \\
1.24 \\
1.62\end{array}$ & $\begin{array}{l}.82 \\
.76 \\
.64\end{array}$ & $\begin{array}{l}1.40 \\
1.33 \\
1.66\end{array}$ & $\begin{array}{l}.56 \\
.57 \\
.86\end{array}$ & $\begin{array}{l}1.16^{*} \\
1.23 \\
1.53\end{array}$ & $\begin{array}{l}.65 \\
.63 \\
.93\end{array}$ \\
\hline Mean & 2.14 & & 2.05 & & 1.40 & & 1.34 & & 1.46 & & 1.31 & \\
\hline
\end{tabular}

Note-Sequences are shown as either $00 \& 01$ for trials following zero, for omission, or 10 \& 11 for trials following unity, for presentation. There were 7-12 experimental series in each condition per observer. $\quad$ *Significantly different from its counterpart of 1.40 $(p<.05, t$ test $)$.

Table 3

Local Detection of Contrast Flashes

\begin{tabular}{|c|c|c|c|c|c|c|c|}
\hline \multirow[b]{2}{*}{ Locus* } & \multicolumn{2}{|c|}{$\mathrm{d}_{\mathrm{R}}^{\prime}$} & \multicolumn{2}{|c|}{$\mathrm{d}_{\mathrm{L}}$} & \multicolumn{2}{|c|}{$\mathrm{d}^{\prime}{ }_{B}$} & \multirow[b]{2}{*}{$\mathrm{BI} \dagger$} \\
\hline & Mean & SEM & Mean & SEM & Mean & SEM & \\
\hline 1 (Left) & 1.37 & .16 & 1.20 & .14 & 2.57 & .16 & 2.00 \\
\hline 2 (Above) & 1.14 & .14 & 1.31 & .14 & 2.50 & .15 & 2.04 \\
\hline 3 (Right) & 1.20 & .16 & 1.49 & .15 & 2.45 & .14 & 1.82 \\
\hline 4 (Below) & 1.26 & .15 & .86 & .11 & 2.19 & .16 & 2.07 \\
\hline Mean & 1.24 & & 1.22 & & 2.43 & & 1.97 \\
\hline
\end{tabular}

Note-Each local mean is based on 28 experimental series, 160 trials per series. Data for the three observers were pooled to arrive at the values in the table. Relative to fixation. +Binocular improvement, obtained as follows: $d_{B}^{\prime} /\left[1 / 2\left(d^{\prime}{ }_{R}+d^{\prime} L\right)\right]$.

ocular performance: $d_{M}^{\prime}=\left[\left(d_{R}^{\prime}+d_{L}^{\prime}\right) / 2\right]$ was similar for all observers. Averaged across observers and all contrast values, mean binocular $\mathrm{d}^{\prime}$ is a factor of 2 larger than mean monocular $\mathrm{d}^{\prime}$, as shown in Table 3 .

As illustrated in Figure 4, mean binocular d' increased fairly linearly with test contrast. Monocular detection was less clearly related to test contrast, and regression slopes are somewhat different for the two eyes, even in observers whose left- and right-eye thresholds were very similar (cf. Table 1).

Figure 5 shows our main result: The advantage obtained in binocular presentation, over monocular presentation, decreases as test contrast increases. The presentation of results in Figure 5 follows Kristofferson's format (in Green \& Swets, 1966, p. 247). For a given test contrast, $d^{\prime}$ values obtained for each locus of stimulation in right and left monocular conditions were summed to estimate predicted binocular detection, on the abscissa. The ordinate plots the values of $d^{\prime}{ }_{B}$ actually obtained. Each point in Figure 5 is thus derived from the results obtained in one binocular series and two monocular series with all three series run at the same test contrast. The line of unity slope represents the locus of all points that satisfy the equation of simple summation. Note that on the left-hand side (poor monocular detection: pre- dicted $d^{\prime}{ }_{B}<2.0$; low test contrast), the prediction of the simple summation model underestimates binocular performance. As monocular performance improves, with higher test contrast, the prediction of simple summation becomes more nearly correct. At still higher test contrasts, at which predicted $3<\mathrm{d}_{\mathrm{B}}^{\prime}$ $<3.6$, binocular performance falls below that predicted by simple summation. The broken line in Figure 5 shows the best-fitting regression line obtained by the least squares method. The line has a .54 slope, has a +1.1 intercept, and accounts for $74 \%$ of the variance in the data. ${ }^{3}$

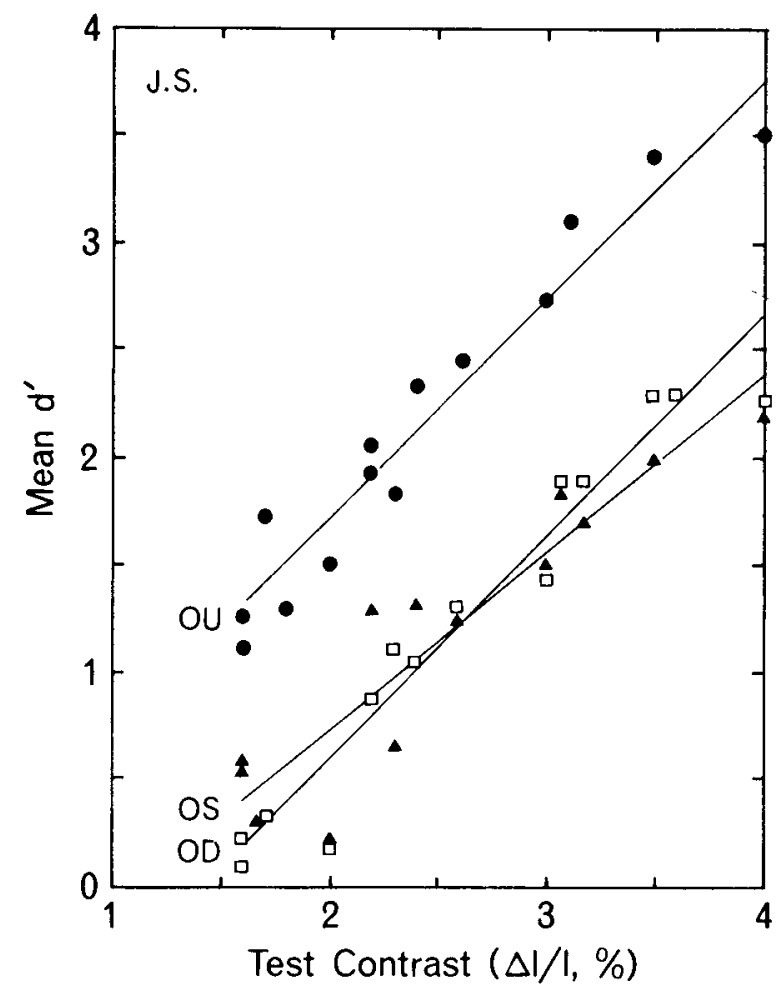

Figure 4. Detection (d', ordinate) as a function of test contrast (abscissa). Interrupted lines are regression best-fitting lines for binocular and monocular detection. Observer J.S. 


\section{Discussion}

Our measurements of $\mathrm{d}^{\prime}$ show that the simple summation model gives a reasonably accurate description of the average binocular improvements in detection of contrast flashes. Yet, regression of the data in Figure 5 suggests that the adequacy of this summation model varies strongly with the stimulus contrast at which measurements are made. At lower values of test contrast, binocular detection is considerably in excess of the sum of monocular d' values; however, at higher test contrast, binocular improvement falls below the prediction of full summation. ${ }^{4}$ Kristofferson (cited in Green \& Swets, 1966) also found decreased summation as flash energy increased: with higher predicted $\mathrm{d}^{\prime}$ values, more of his experimental points tended to fall below the line of full summation (see Green \& Swets, 1966, Figure 9-5b, p. 247). This same trend is even more pronounced in our data.

If the binocular output is always produced by an addition rule that sums monocular inputs (the model of energy summation) or monocular outputs (the models of square-root summation and probability summation), then the drop in the amount of binocular advantage with increasing strength of monocular

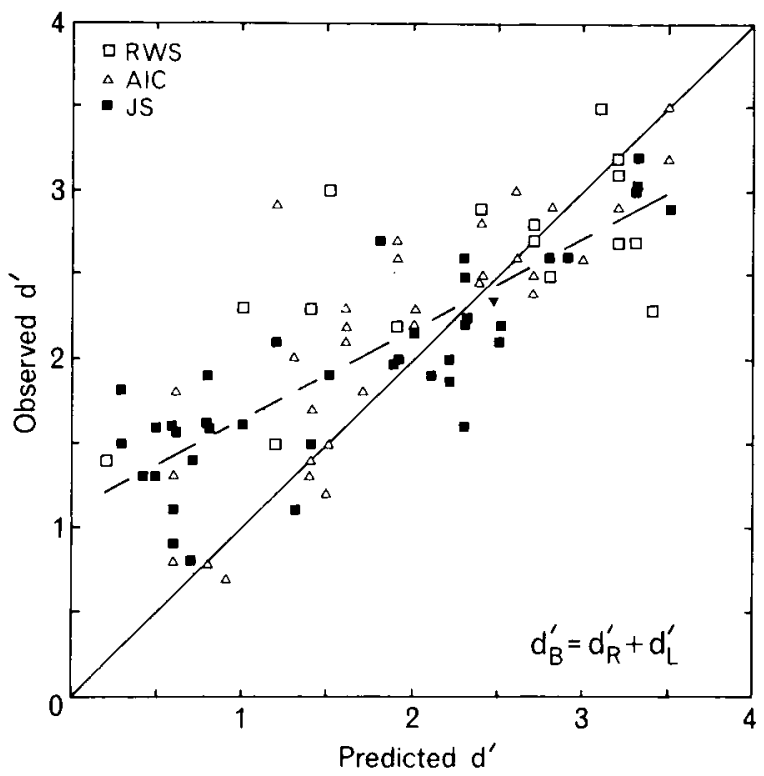

Figure 5. Binocular improvement in detection of contrast flashes. Observed $d^{\prime}{ }_{B}$ values (ordinate) plotted as a function of prediction generated by simple summation of monocular $d^{\prime}$ values (abscissa). For each point, one coordinate was produced by adding up the two monocular d' values, obtained for a given stimulation locus, at a particular contrast, and the other coordinate given by the binocular d' value found for the same locus at the same test contrast. [This figure shows the distribution of the 87 experimental points with predicted $d_{B}^{\prime}<3.6$; excluded are 26 experimental points with mean predicted $d_{B}^{\prime}=4.43$ (SEM $\left.=.10\right)$, corresponding to mean observed $d^{\prime}=3.36(\mathrm{SEM}=.04)$. The artificial "ceiling" of the $d^{\prime}$ measure reached when $d_{B}^{\prime}$ exceeds 3.5 (Swets, 1964) makes it impractical to check predictions of binocular improvement when average monocular $d^{\prime}$ exceeds 1.75$]$. stimulation is hard to understand. The model in Figure 1c provides an explanation for this result. According to the model, addition occurs for matched monocular inputs. These are fused to form an exclusively binocular channel. Unmatched inputs go their separate monocular routes. However, at the second stage, the signal from either monocular channel can interact with the signal generated by the binocular channel. In the net response that is the basis for the perception of the contrast flash, the unmatched monocular response can be attenuated by the binocular response. The magnitude of this attenuation should be proportional to binocular background luminance summation, but it must also vary with the strength of the monocular response. Presumably, weak unmatched input would not interfere with the binocular channel appreciably. (Thus, weak monocular flashes were not detected because they appeared against a fused, i.e., binocularly summed, luminous background.) As the monocular flashes increase in energy, they tend to interrupt binocular summation of background luminance, and thus noticeably affect the net response. As the (suprathreshold) flashes get stronger, detection depends more and more on the adaptation state in the stimulated monocular channel alone (Cogan, 1982). Of course, in the binocular condition, all input was matched and the increments, whether strong or weak, were summed in the same fused channel as the background.

\section{EXPERIMENT 2: MONOCULAR DETECTION OF CONTRAST FLASHES IS IMPROVED WHEN A GANZFELD IS VIEWED BY THE OPPOSITE EYE}

The preceding discussion implies that monocular sensitivity may have been significantly underestimated in the "monocular" condition of Experiment 1 . The magnitude of underestimation should be approximately proportional to the amount of binocular summation of background luminances occurring during binocular fusion of contours. Detectability of monocularly presented contrast flashes should improve, and the binocular advantage should decrease, if binocular summation of background luminances is prevented during monocular detection.

To falsify this proposition, it should be sufficient to eliminate all fusable contours in the companion eye while keeping the mean luminance of both monocular fields the same. One way to accomplish this is by introducing a Ganzfeld that is not expected to fuse with contralateral contours. Detectability of contrast flashes in the eye contralateral to the Ganzfeld would now depend on the sensitivity of this eye alone, and therefore should be higher than in the "monocular" condition of Experiment 1 (in which background contours were binocularly fused).

Three conditions were tested. The monocular base- 
line condition (M-baseline) measured the visibility of test flashes delivered to one eye while a luminous Ganzfeld was presented to the other eye. A second condition, binocular baseline (B-baseline) was identical to that used previously (targets and stimuli in register to both eyes). In the third condition, monocular test (M-test), the background targets were fused binocularly, but the test stimuli were given to only one eye. This last condition, of course, is identical to the "monocular" condition of Experiment 1. In all conditions, the observer had to detect and localize contrast flashes, as before.

\section{Method}

Apparatus. The apparatus was the same except that in the Mbaseline condition a diffusing screen was placed $12 \mathrm{~mm}$ in front of the nontested eye; the screen was illuminated by a separate tungsten source such that its luminance, at any point in the field within at least $90 \mathrm{deg}$ from the center, was equal to the mean luminance of the background field presented to the tested eye.

Procedure. Stimulus presentation was the same as in Experiment 1 . The experiment began and ended with the B-baseline condition. The M-baseline and M-test conditions were run in a pseudorandom sequence counterbalanced for the right and left eyes. Background luminance was $100 \mathrm{~cd} / \mathrm{m}^{2}$ in all conditions, for all observers. Flash energies and corresponding test contrasts were selected for each observer to allow all three observers to perform similarly in the series run first (the B-baseline condition). To achieve this, we adjusted the steady state luminance of the LEDs producing test contrasts ( $\Delta \mathrm{I} / \mathrm{I})$ equal to $3.7 \%, 4.5 \%$, and $6.0 \%$ for observers J.S., A.I.C., and R.W.S., respectively (based on threshold estimates, see General Procedure section). For each observer, each of the three conditions was run twice. Measurements for any one observer were done during some $2.5 \mathrm{~h}$ on the same day.

\section{Results}

As expected, detection in the $\mathrm{M}$-baseline condition (mean $\mathrm{d}^{\prime}=1.83, \mathrm{SEM}=.12$ ) was better than in the M-test condition $\left(\mathrm{d}^{\prime}=1.13, \mathrm{SEM}=.10\right)$, an average of $62 \%$ improvement. This is an estimate of the effect of binocular summation of the background luminance upon monocular detection of contrast flashes, as given by the comparison M-baseline/M-test [1.83/ $1.13=1.62$ ]. As Table 4 shows, there is significant variability in the magnitude of the effect for right and left eye of all observers. The overall differences among the three conditions are highly significant $[F(2,15)=15.66 ; \mathrm{p}<.01]$.

Performance was best in the B-baseline condition (mean $\mathrm{d}^{\prime}=2.59, \mathrm{SEM}=.04$ ). Note that the B-baseline results are similar for all observers and for the series run first and last. (This means that the contrast was scaled appropriately and that individual sensitivity probably remained stable throughout the experiment.) In the M-baseline condition, the eyes performed very similarly in two observers (A.I.C. and J.S.), while the left eye of observer R.W.S. performed more than twice as well as his right eye. Relative performance of the fused binocular channel and a given monocular channel obtains as the ratio of $\mathrm{d}^{\prime}$ values for the comparison B-baseline/M-baseline. On the average, binocular performance was $41.5 \%$ better $\left[\mathrm{d}^{\prime}{ }_{\mathrm{B}} / \mathrm{d}^{\prime}{ }_{\mathrm{M}}=2.59 / 1.83=1.415\right]$. But the amount of binocular improvement varied greatly among observers: $16.5 \%$ in A.I.C., $35 \%$ in J.S., and $132 \%$ in R.W.S. In the last case, the average value of $132 \%$ may be misleading, because R.W.S. showed a strong difference between the ratios for each eye: over his left eye, the binocular improvement was $42 \%$; over his right eye, the binocular improvement was $222 \%$.

A comparison of detection in the B-baseline and M-test conditions reveals a remarkable similarity in the outcomes of Experiments 1 and 2; at about the same test contrast (the closest values used in both experiments), performance was very nearly the same for all observers (Table 5).

\section{Discussion}

Eliminating all contours in the nontested eye in the M-baseline condition improved monocular detection of contrast flashes an average of $62 \%$. Thus, monocular sensitivity is better when the background viewed by the opposite eye is a homogeneous luminous field than when the background contains contours matching those in the tested eye. This result was expected on the assumption that binocularly matched background contours fuse, permitting background luminances to sum. Under such conditions, a

Table 4

Mean Detection (d') in Different Conditions

\begin{tabular}{|c|c|c|c|c|c|c|c|c|c|c|c|c|}
\hline \multirow[b]{4}{*}{ Observer } & \multicolumn{12}{|c|}{ Experimental Condition } \\
\hline & \multicolumn{4}{|c|}{$\begin{array}{c}\text { Binocular Baseline } \\
\left(\text { Mean } \mathrm{d}^{\prime}=2.59, \mathrm{SEM}=.04 ; \mathrm{n}=24\right)\end{array}$} & \multicolumn{4}{|c|}{$\begin{array}{c}\text { Monocular Baseline } \\
\left(\text { Mean } \mathrm{d}^{\prime}=1.83, \mathrm{SEM}=.12 ; \mathrm{n}=24\right)\end{array}$} & \multicolumn{4}{|c|}{$\begin{array}{c}\text { Monocular Test } \\
\left.\text { (Mean } \mathrm{d}^{\prime}=1.13, \mathrm{SEM}=.10 ; \mathrm{n}=24\right)\end{array}$} \\
\hline & \multicolumn{2}{|c|}{1} & \multicolumn{2}{|c|}{2} & \multicolumn{2}{|c|}{ Right Eye } & \multicolumn{2}{|c|}{ Left Eye } & \multicolumn{2}{|c|}{ Right Eye } & \multicolumn{2}{|c|}{ Left Eye } \\
\hline & Mean & SD & Mean & SD & Mean & SD & Mean & SD & Mean & SD & Mean & SD \\
\hline J.S. & 2.64 & .32 & 2.70 & .37 & 2.06 & .42 & 1.91 & .53 & 1.10 & .54 & 1.60 & .44 \\
\hline A.I.C. & 2.80 & .32 & 2.51 & .26 & 2.26 & .24 & 2.28 & .58 & 1.76 & .51 & 1.12 & .24 \\
\hline R.W.S. & 2.64 & .67 & 2.26 & .41 & .76 & .44 & 1.73 & .69 & .41 & .28 & .81 & .34 \\
\hline
\end{tabular}

Note-To obtain mean d' values and the standard error of the mean (SEM), we collapsed the d' across the four tested loci, the eyes, and the observers giving the 24 scores for each mean. 
Table 5

Comparison of Detection at Similar Contrast in Experiments 1 and 2

\begin{tabular}{|c|c|c|c|c|c|c|}
\hline \multirow[b]{2}{*}{ Observer } & \multirow[b]{2}{*}{ Experiment } & \multirow{2}{*}{$\begin{array}{c}\text { Test } \\
\text { Contrast* }\end{array}$} & \multicolumn{3}{|c|}{ Detection } & \multirow{2}{*}{$\begin{array}{c}\text { Binocular } \\
\text { Improvement } \dagger\end{array}$} \\
\hline & & & $\mathrm{d}_{\mathrm{R}}^{\prime}$ & $\mathrm{d}_{\mathrm{L}}^{\prime}$ & $\mathrm{d}_{\mathrm{B}}^{\prime}$ & \\
\hline $\begin{array}{l}\text { J.S. } \\
\text { J.S. }\end{array}$ & $\begin{array}{l}1 \\
2\end{array}$ & $\begin{array}{l}3.0 \\
3.7\end{array}$ & $\begin{array}{l}1.42 \\
1.10\end{array}$ & $\begin{array}{l}1.50 \\
1.60\end{array}$ & $\begin{array}{l}2.74 \\
2.67\end{array}$ & $\begin{array}{l}1.88 \\
1.98\end{array}$ \\
\hline $\begin{array}{l}\text { A.I.C. } \\
\text { A.I.C. }\end{array}$ & $\begin{array}{l}1 \\
2\end{array}$ & $\begin{array}{l}4.0 \\
4.5\end{array}$ & $\begin{array}{l}1.33 \\
1.76\end{array}$ & $\begin{array}{l}1.49 \\
1.12\end{array}$ & $\begin{array}{l}2.70 \\
2.66\end{array}$ & $\begin{array}{l}1.92 \\
1.85\end{array}$ \\
\hline $\begin{array}{l}\text { R.W.S. } \\
\text { R.W.S. }\end{array}$ & $\begin{array}{l}1 \\
2 \\
\end{array}$ & $\begin{array}{l}5.6 \\
6.0\end{array}$ & $\begin{array}{l}.42 \\
.41 \\
\end{array}$ & $\begin{array}{l}.58 \\
.81 \\
\end{array}$ & $\begin{array}{l}2.26 \\
2.45\end{array}$ & $\begin{array}{l}4.52 \\
4.00\end{array}$ \\
\hline
\end{tabular}

Note- $d^{\prime}{ }_{R}$ and $d^{\prime}{ }_{L}$ measure detection in the fused condition, the test flash given to one eye, right or left, respectively; $d^{\prime}{ }_{B}$ measures detection in the fully fused condition, the flash given to both eyes simultaneously on corresponding retinal areas. $A$ year elapsed between Experiments 1 and 2. ${ }^{*} \Delta I / I$, in percent. $\left.\quad{ }^{\prime} d_{B}^{\prime} /\left(d_{R}^{\prime}+d_{\mathrm{L}}^{\prime}\right) / 2\right]$.

monocular luminance increment must be detected against a higher effective adaptation state, making its detection more difficult.

The comparison of contrast detection in the fully fused condition (B-baseline) and the "purely monocular" condition (M-baseline) produced very different individual amounts of binocular improvement, ranging from $16 \%$ (A.I.C., left eye) to $222 \%$ (R.W.S., right eye). In the face of such differences, the mean value of $41.5 \%$ improvement in binocular detection over monocular detection has little meaning. But it is important to realize that the variability does not invalidate the approach. We are, indeed, comparing contrast detection in two different channels: the fused binocular channel and a separate monocular channel. When contrast detection in the M-baseline condition is the same for both eyes of an observer, then the monocular baseline will be accurately given by the average of monocular d's. This was very nearly the case with Observers A.I.C and J.S. What should be now expected for detection in binocular observation keeping the luminance, the increments, and the spatial relations unchanged? If full energy summation takes place, then the contrast threshold must remain the same. The increment is summed, but so is the background. Obviously, contrast at threshold should not change. But this is not what actually happens: the threshold goes down in binocular observation. This result implies that summation is somehow more effective for the increment than for the background. In other words, the binocular channel must be functionally different from a monocular channel, at least as far as detection of contrast flashes is concerned. This must be true whatever the magnitude of binocular improvement in contrast detection.

\section{GENERAL DISCUSSION}

As Thorn and Boynton (1974) have noted, the magnitude of binocular advantage over monocular performance does not tell us much about the underlying processes unless the baseline has been anchored to a clearly specified physiological mechanism. Where increment thresholds are concerned, monocular contrast sensitivity depends on the adaptation state. At least within the limits of Weber's law $(\Delta \mathrm{I} / \mathrm{I}=$ const $)$, changes in the adaptation state are a linear function of luminance (I). But the Weber fraction depends nonlinearly on spatial interactions as well. Thus, a gradual increase in the size of the luminous background will first depress and then enhance sensitivity for a point increment at the center of the background (Westheimer, 1965, 1967). Westheimer has interpreted the phenomenon in terms of receptive field size and organization, with lateral inhibition as the main mechanism. It is possible that the higher contrast sensitivity of the binocular channel is due to a similar mechanism operating in the receptive field of exclusively binocular cells that respond only to matched binocular contours. Hubel and Wiesel (1970) have demonstrated that most of the "depth cells" in area 18 of the macaque monkey respond briskly to simultaneous and corresponding stimuli from two eyes, but fail to respond to stimulation of either eye alone. This may be the neural substratum for the fused channel.

The case of a monocular luminance increment presented against a binocularly fused background the monocular condition of Experiment 1, identical to the monocular test in Experiment 2) is particularly important for the model under study. As indicated in Figure 1c, the model proposes that the perception of a monocular contrast flash depends upon the net response of two channels: the fused binocular channel and one monocular channel. In a trivial sense, it is obvious that monocularly presented contours can be easily incorporated in the binocular visual field (e.g., the nonius marks signaling alignment of the eyes during binocular fusion). Experiments described in this paper suggest that the channel that transmits fused contours and the interocular channel that processes unmatched monocular contours keep their separate identities and interact before the "inclusive" output is produced.

The model in Figure 1c is not the first in which two 
channels or mechanisms were postulated to account for observations concerned with binocular detection of flashed luminance increments. In particular, Cohn and Lasley (1976) have proposed a mathematical model for the operation of two independent binocular mechanisms, one that sums signals from the two eyes and one that computes the difference, before the decision is reached by a common center. While the present paper was in revision, an updated version of this model was published (Cohn, Leong, \& Lasley, 1981). As we see it, the main difference between the Cohn-Lasley model and the model in Figure 1c is that our model explicitly separates the cases of fusion and nonfusion. All available evidence clearly indicates that fusion does present a special case. In our model, the contribution of the fused binocular channel to the net response is probably mediated by a mechanism of the AND-gate type, while the interocular channel produces its (monocular) contribution to the net response through a mechanism operating as an exclusive OR gate. We do not see evidence for a mechanism that would accept unmatched signals from both eyes and perform a straightforward difference computation. Note that none of the existing models has a mechanism to describe the operation of the hypothetical common decision center that produces the inclusive binocular output upon interaction between the binocular channel and the interocular channel.

\section{REFERENCES}

Apkarian, P. A., Nakayama, K., \& TYler, C. W. Binocularity in the human visual evoked potential: Facilitation, summation and suppression. Electroencephalography and Clinical Neurophysiology, 1981, 51, 32-48.

Blake, R., \& Fox, R. The psychophysical inquiry into binocular summation. Perception \& Psychophysics, 1973, 14, 161-185.

Bouman, M. A. On foveal and peripheral interaction in binocular vision. Optica Acta, 1955, 1, 177-183.

Burns, B. D., \& Pritchard, R. Cortical conditions for fused binocular vision. Journal of Physiology (London), 1968, 197, 149-171.

Campbell, F. W., \& Green, D. G. Monocular versus binocular visual acuity. Nature, 1965, 208, 191-192.

Cogan, A. I. Monocular sensitivity during binocular viewing. Vision Research, 1982, 22, 1-16.

Cohn, T. E., \& LAsley, D. J. Binocular vision: Two possible central interactions between signals from two eyes. Science, 1976, 192, 561-563.

Cohn, T. E., Leong, H., \& Lasley, D. J. Binocular luminance detection: Availability of more than one central interaction. Vision Research, 1981, 21, 1017-1023.

Collter, G. Probability of response and internal association as functions of monocular and binocular stimulation. Journal of Experimental Psychology, 1954, 47, 75-83.

Crawford, B. H. Ocular interaction in its relation to measurements of brightness threshold. Proceedings of the Royal Society $B, 1940,128,552-559$.

Crozien, W. J., \& Holway, A. H. Theory and measurement of visual mechanisms. I. A visual discriminometer. II. Threshold stimulus intensity and retinal position. Journal of General Physiology, 1938, 22, 341-364.

Crozier, W. J., \& Holway, A. H. Theory and measurement of visual mechanisms. III. $\Delta \mathrm{I}$ as a function of area, intensity, and wavelength, for monocular and binocular stimulation. Journal of General Physiology, 1939, 23, 101-141.

Green, D. M., \& Swets, J. A. Signal detection theory and psychophysics. Huntington, N.Y: Krieger, 1966.

HubeL, D. H., \& WIESEL, T. N. Stereoscopic vision in macaque monkey. Nature, 1970, 225, 41-42.

LEGGE, G. E., \& RUBin, G. S. Binocular interactions in suprathreshold contrast perception. Perception \& Psychophysics, $1981,30,49-61$.

Matin, L. Binocular summation at the absolute threshold of peripheral vision. Journal of the Optical Society of America, 1962, 52, 1276-1286.

Nelson, J. I., Kato, H., \& Bishop, P. O. Discrimination of orientation and position disparities by binocularly activated neurons in cat striate cortex. Journal of Neurophysiology, 1977, 40, 260-283.

Pirenne, M. H. Binocular and uniocular threshold of vision. Nature, 1943, 152, 698-699.

SWETs, J. A. Signal detection and recognition by human observers. New York: Wiley, 1964.

Thorn, F., \& Boynton, R. M. Human binocular summation at absolute threshold. Vision Research, 1974, 14, 445-458.

WESTENDORF, D. H., \& Fox, R. Binocular detection of disparate light flashes. Vision Research, 1977, 17, 697-702.

Westheimer, G. Spatial interaction in the human retina during scotopic vision. Journal of Physiology (London), 1965, 181, 881-894.

WESTHEIMER, G. Spatial interaction in human cone vision. Journal of Physiology (London), 1967, 190, 139-154.

\section{NOTES}

1. Binocular facilitation has been found for the human visual evoked potential (cf. Apkarian, Nakayama, \& Tyler, 1981).

2. Since monocular outputs have to be combined at some point before the observer's decision is made, all models which assume binocular improvement in performance are models of physiological summation (as distinct, for instance, from a "sociological summation," in which two equally good observers reporting to a common counter could improve upon detection by each observer alone).

3. The best-fitting line, shown in Figure 5, was calculated using the 33 different available $x$ values. The number of observations (N) per $x$ value varied from 1 to 7; when $N$ was larger than 1 , the arithmetic mean was substituted. To check whether the variability in $\mathbf{N}$ could have significantly affected the regression, we made a separate calculation, excluding all points where $\mathrm{N}<4$ and using the 15 values obtained as arithmetic means of four to seven observations. For these 15 values, distributed fairly evenly on the abscissa, the best-fitting line has a slope of .60 , at a y-intercept of 1.06. The correlation coefficient is improved $(\mathrm{r}=.96)$; otherwise, the difference between the two regression lines is quite small.

6. The use of the detectability theory parameter d' (Swets, 1964) gives reliable estimates of detectability when detection is significantly less than perfect. As $d^{\prime}$ approaches the value of 3.5 , there is not much room for improvement in detection. It therefore makes sense to set this limit for $\mathrm{d}_{\mathrm{B}}{ }_{\mathrm{B}}$ when one estimates binocular improvement in detectability.

(Manuscript received May 26, 1981; revision accepted for publication December 3,1981 .) 